\title{
Scoliosis Caused by Limb-Length Discrepancy in Children
}

\author{
Kazuyoshi Kobayashi, Kei Ando, Hiroaki Nakashima, Masaaki Machino, \\ Masayoshi Morozumi, Shunsuke Kanbara, Sadayuki Ito, Taro Inoue, \\ Hidetoshi Yamaguchi, Kenichi Mishima, Naoki Ishiguro, Shiro Imagama \\ Department of Orthopedic Surgery, Nagoya University Graduate School of Medicine, Nagoya, Japan
}

Study Design: Single-center retrospective study in pediatric patients.

Purpose: The purpose of the study was to investigate the effects of the presence and severity of leg-length discrepancy (LLD) on scoliosis in children.

Overview of Literature: LLD is a common orthopedic problem that can result in standing imbalance, low back pain, and stress fractures. It may cause structural changes in the spine over time, but the exact effect of LLD on scoliosis is unknown.

Methods: The subjects were 23 children with LLD $\geq 20 \mathrm{~mm}$ (range, 27-65 mm) treated at Nagoya University Hospital between 2007 and 2017. Whole spine posteroanterior standing radiographs and whole lower limb radiographs in a supine position were recorded. Data were collected for demographics, LLD, Cobb angle, pelvic obliquity, and Nash/Moe index. Scoliosis was defined as a Cobb angle $\geq 10^{\circ}$. Leg length was measured from the top of the femoral head to the middle of the tibial plafond, and LLD was defined as the difference between the left and right leg lengths.

Results: The patients (nine males and 14 females) had a mean age of 14.0 years (range, 5-18 years). The average LLD was $44.3 \pm 17.2$ $\mathrm{mm}$, with LLDs of 20 to $39 \mathrm{~mm}, 40$ to $59 \mathrm{~mm}$, and $\geq 60 \mathrm{~mm}$ in 13, five, and five subjects, respectively. The average Cobb angle was $13.0^{\circ} \pm 7.0^{\circ}$, and 15 subjects (65\%) had scoliosis. Convexity of the scoliosis was to the short leg side in all cases. The Cobb angle was significantly related to the severity of the $\operatorname{LLD}(R=0.736, p<0.01)$, pelvic obliquity $(R=0.966, p<0.01)$, and Nash/Moe index $(p<0.05)$. Conclusions: LLD is a common pediatric condition that can cause scoliosis of the spine. Severe scoliosis may develop if the LLD is $\geq 30 \mathrm{~mm}$. Long-term studies are needed to examine the effect of LLD resolution on the elimination of scoliosis.

Keywords: Scoliosis; Leg length inequality; Child; Cobb angle; Pelvic obliquity

\section{Introduction}

Leg-length discrepancy (LLD) is a common orthopedic problem that can lead to standing imbalance, running injuries, low back pain, stress fractures, and osteoarthritis of the hip [1]. LLD is usually small (about $1 \mathrm{~cm}$ ) and occurs in $3 \%$ to $15 \%$ of the population. It is caused by overgrowth, growth disorder, and dysplasia of the lower limbs, which all lead to a difference in the lengths of the limbs. LLD is divided into congenital and acquired cases. Congenital cases include those in which LLD is clear at birth or becomes apparent with growth. In acquired cases,

Received Dec 5, 2019; Revised Jan 28, 2020; Accepted Feb 18, 2020

Corresponding author: Shiro Imagama

Department of Orthopedic Surgery, Nagoya University Graduate School of Medicine, 65 Tsurumai Showa-ward, Nagoya, Aichi 4668550 , Japan

Tel: +81-52-741-2111, Fax: +81-52-744-2260, E-mail: imagama@med.nagoya-u.ac.jp 
trauma, infection, and tumors may obstruct the epiphyseal growth line and cause LLD [2-7].

The major skeletal reactions or adaptations to LLD are pelvic obliquity and scoliosis [8]. Scoliosis due to LLD is referred to as functional scoliosis, and it totally or partially regresses when the LLD is removed [9]. The pattern of scoliosis associated with LLD is described as compensatory, nonstructural, and non-progressive $[10,11]$, but it has been suggested that LLD could produce structural changes in the spine with time [10]. LLD can also occur secondary to scoliosis, particularly in the case of compensatory scoliosis. In these cases, the LLD appears as the result of an asymmetrical load on the lower extremities $[12,13]$. However, the details of the variations of LLD and its relationship with pelvic obliquity are unknown. Therefore, the purpose of this study was to investigate scoliosis associated with LLD in children without other diseases related to the spine and to examine the correlations between scoliosis and the cause and severity of LLD and pelvic obliquity.

\section{Materials and Methods}

The subjects were 23 children with LLD $\geq 20 \mathrm{~mm}$ (range, 27-65 mm) treated at Nagoya University Hospital between 2007 and 2017. Patients with previous spinal surgery for scoliosis or with an LLD $<20 \mathrm{~mm}$ were excluded. The underlying diseases in the patients were hemihypertrophy $(n=5)$, congenital shortening $(n=4)$, posttraumatic fracture $(n=3)$, Perthes disease $(n=3)$, congenital hip dislocation $(n=3)$, lower limb bone tumor $(n=2)$, hemihypoplasia $(n=2)$, and congenital constriction band $(n=1)$. The patients included nine males and 14 females with a mean age of 14.0 years (range, 5-18 years) at the time of examination. The study was approved by the Ethics Committee of Nagoya University Hospital (IRB approval no., 354-3). Informed consent was obtained from all individual participants included in the study.

Whole spine posteroanterior (PA) standing radiographs and whole lower limb radiographs in a supine position were recorded for all patients. Radiographs were obtained without a brace. The following data were collected: demographics (age, height, weight, sex), LLD, and radiographic parameters (major curve Cobb angle, pelvic obliquity, Nash/Moe index). Scoliosis was defined as a coronal Cobb angle $\geq 10^{\circ}$ [14]. Radiographic measurements were performed twice, and the average was used in the pri- mary analysis. Radiographs were taken with the hips and knees extended and the pelvis not rotated. Leg length was measured from the top of the femoral head to the middle of the tibial plafond. The difference between the left and right leg length was defined as the LLD. The Cobb angle was measured as the angle between the upper and lower vertebrae angle [15]. Pelvic obliquity was defined as the angle between the horizontal and pelvic coronal reference lines [16]. Axial rotation of the apical vertebrae was assessed on PA radiographs to define the Nash/Moe index [17].

Differences between two groups divided by the Nash/ Moe index in relation to the axial rotation of the apical vertebrae were analyzed by Student $t$-test. A post hoc Bonferroni test was used to assess the significance of differences of one group from all other groups. A Pearson correlation coefficient was calculated for the correlation between the Cobb angle and LLD and between the Cobb angle and pelvic obliquity. Receiver operating characteristic (ROC) curves were drawn to determine the cutoff LLD, and the sensitivity and specificity at the optimal cutoff were calculated. All analyses were conducted using IBM SPSS ver. 22.0 for Windows (IBM Corp., Armonk, NY, USA). All $p<0.05$ were considered to be significant in all analyses.

\section{Results}

Demographic data for the 23 subjects are shown in Table 1. The average LLD was $44.3 \pm 17.2 \mathrm{~mm}$, and LLDs of 20 to $39 \mathrm{~mm}, 40$ to $59 \mathrm{~mm}$, and $\geq 60 \mathrm{~mm}$ were present in 13 , five, and five subjects, respectively (Table 1). The Cobb angle, pelvic obliquity, and Nash/Moe index of the apex vertebra are shown in Table 2. The average Cobb angle was $13.0^{\circ} \pm 7.0^{\circ}$, and 15 subjects (65\%) had scoliosis (Cobb angle $\geq 10^{\circ}$ ), including four with a Cobb angle $\geq 20^{\circ}$. A lumbar scoliosis curve was present in 20 cases (87\%). Convexity of the scoliosis was to the short leg side in all cases. The Nash/Moe index was grade 0 in 16 subjects and grade 1 in seven subjects.

The Cobb angle was significantly related to the severity of the LLD $(R=0.736, p<0.01)$ (Fig. 1), and more severe scoliosis occurred at an LLD $\geq 30 \mathrm{~mm}$. There were also significant associations of the Cobb angle with pelvic obliquity $(R=0.966, p<0.01)$ (Fig. 2$)$ and the Nash/Moe index (Fig. 3). Details of the Cobb angle, pelvic obliquity, and Nash/Moe index classified by LLD are shown in Table 
Table 1. Demographic and clinical data for patients with LLD ( $\mathrm{n}=23)$

\begin{tabular}{lc}
\hline Characteristic & Value \\
Female & $14(61)$ \\
\hline Age $(\mathrm{yr})$ & $14.0 \pm 2.9$ \\
\hline Height $(\mathrm{cm})$ & $147.5 \pm 17.8$ \\
\hline Weight $(\mathrm{kg})$ & $43.0 \pm 13.3$ \\
\hline Body mass index $\left(\mathrm{kg} / \mathrm{m}^{2}\right)$ & $19.1 \pm 2.8$ \\
\hline LLD (mm) & \\
\hline $20-39$ & $13(56)$ \\
\hline $40-59$ & $5(22)$ \\
\hline$\geq 60$ & $5(22)$ \\
\hline Average & $44.3 \pm 17.2$ \\
\hline Etiology & \\
\hline Hemihypertrophy & $5(22)$ \\
\hline Congenital shortening & $4(17)$ \\
\hline Post traumatic fracture & $3(13)$ \\
\hline Perthes disease & $3(13)$ \\
\hline Congenital hip dislocation & $3(13)$ \\
\hline Lower limb bone tumor & $2(9)$ \\
\hline Hemihypoplasia & $2(9)$ \\
\hline Congenital fibular hemimelia & $1(4)$ \\
\hline
\end{tabular}

Values are presented as number (\%) or mean \pm standard deviation. LLD, leg-length discrepancy.

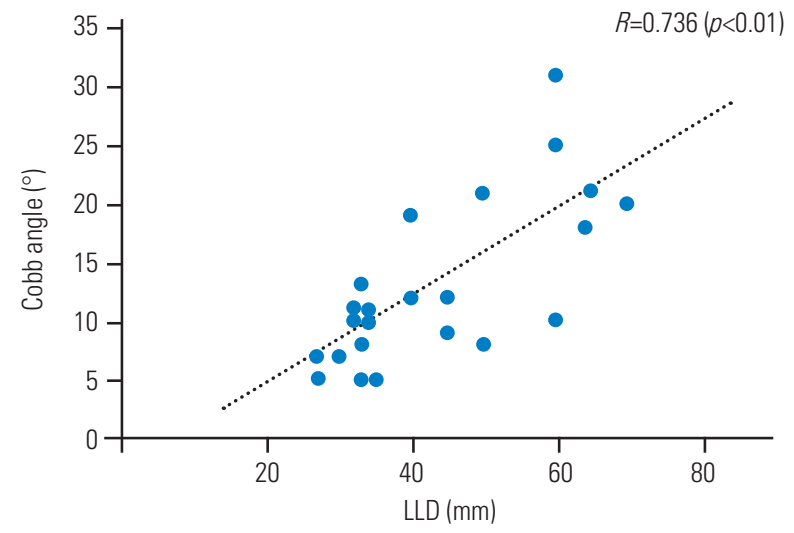

Fig. 1. The Cobb angle had a significant positive correlation with the severity of $\operatorname{LLD}(R=0.736, p<0.01)$. LLD, leg-length discrepancy.

3. Significant differences were found in all cases with LLD $\geq 60 \mathrm{~mm}$ (Table 3). An ROC curve to identify an LLD cutoff value $(\mathrm{mm})$ for the prediction of scoliosis $\left(\geq 10^{\circ}\right)$ is shown in Fig. 4. Two typical cases are described in the following paragraphs:

Case 1: A 13-year-old female with left femoral hypoplasia and LLD $(51 \mathrm{~mm})$ shown in lower limb radiography
Table 2. Cobb angle, pelvic obliquity, and Nash/Moe index in patients with leg-length discrepancy $(\mathrm{n}=23)$

\begin{tabular}{lc} 
Variable & Value \\
Major curve Cobb angle $\left(^{\circ}\right)$ & $8(35)$ \\
$<10$ & $11(48)$ \\
$10-19$ & $3(13)$ \\
$20-29$ & $1(4)$ \\
\hline$\geq 30$ & $13.0 \pm 7.0$ \\
\hline Average & \\
\hline Pelvic obliquity $\left(^{\circ}\right)$ & $16(35)$ \\
\hline$<10$ & $6(48)$ \\
\hline $10-19$ & $1(13)$ \\
\hline 20 & $9.0 \pm 6.3$ \\
\hline Average & \\
\hline Nash/Moe index of the apex vertebra & $16(70)$ \\
\hline 0 & $7(30)$ \\
\hline 1 & 0 \\
\hline$\geq 2$ & $20(87)$ \\
\hline Apex of the scoliosis & \\
\hline Thoracic & \\
\hline Lumbar & \\
\hline
\end{tabular}

Values are presented as number (\%) or mean \pm standard deviation.

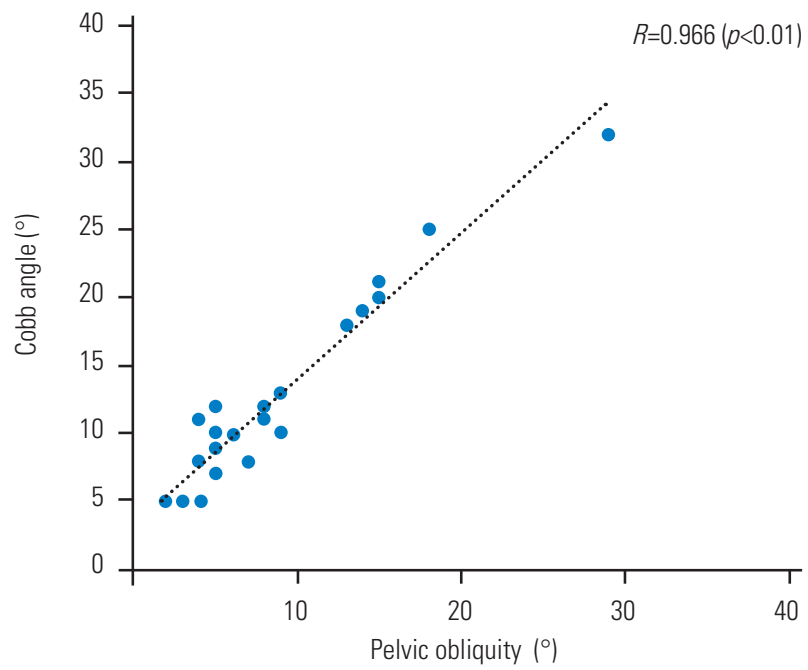

Fig. 2. The Cobb angle had a significant positive correlation with pelvic obliquity $(R=0.966, p<0.01)$.

in a supine position (Fig. 5A). In a whole spine PA radiograph on standing, pelvic obliquity was $16^{\circ}$, the Cobb angle was $21^{\circ}$ at Th12-L5, and the Nash/Moe index was 1 (Fig. 5B).

Case 2: An 8-year-old female with congenital fibular hemimelia and LLD $(60 \mathrm{~mm})$ shown in lower limb radi- 


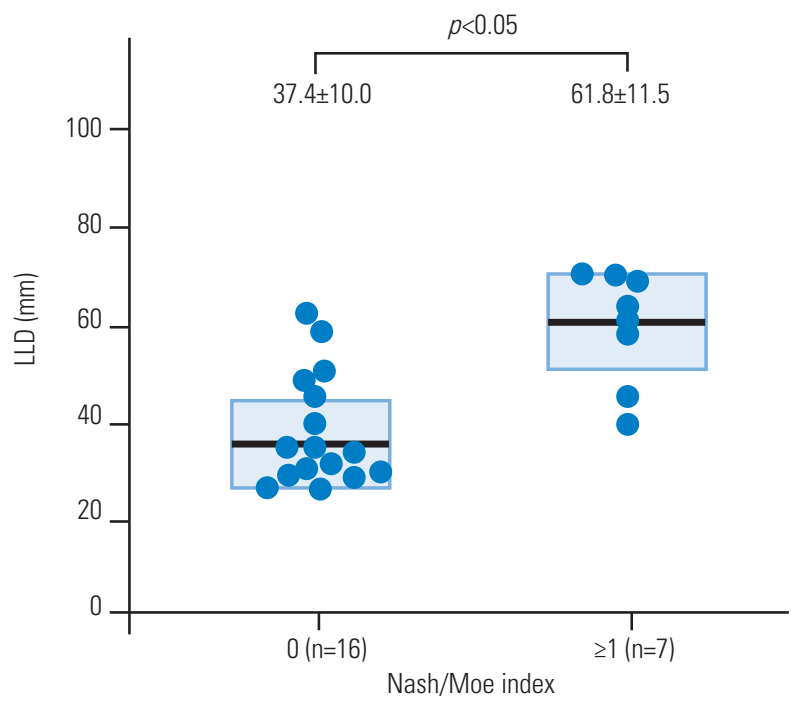

Fig. 3. LLD differed significantly between patients with a Nash/Moe index of 0 and $\geq 1$ ( $p<0.05)$. LLD, leg-length discrepancy.

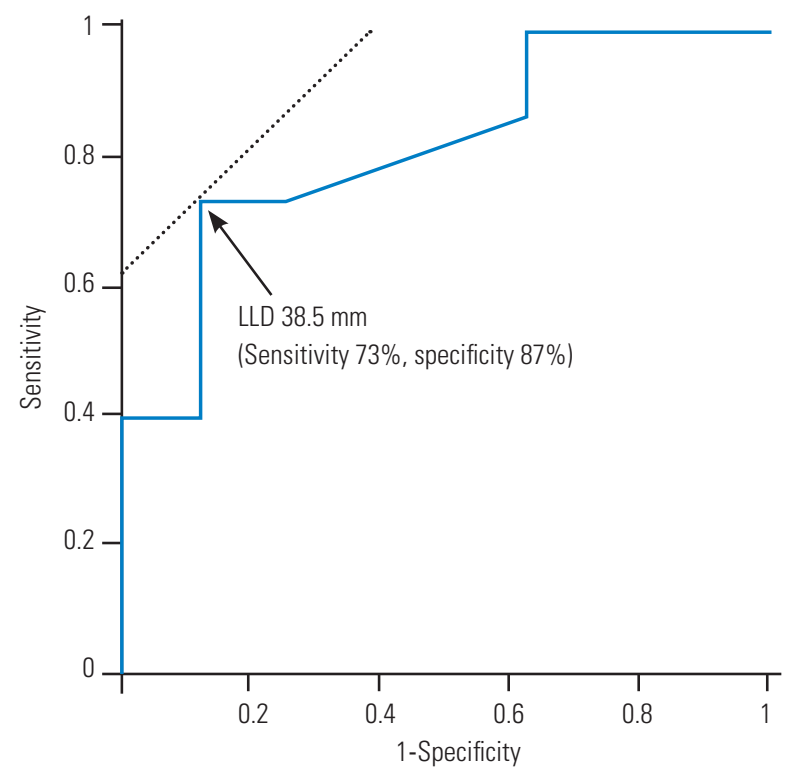

Fig. 4. Receiver operating characteristic curves were used to determine the cutoff LLD $(\mathrm{mm})$ to predict scoliosis. LLD, leg-length discrepancy. ography in a supine position. She had a history of surgery to the lower extremity (Fig. 6A). In a whole spine PA radiograph on standing, pelvic obliquity was $29^{\circ}$, the Cobb angle was $32^{\circ}$ at Th12-L5, and the Nash/Moe index was 1 (Fig. 6B).

\section{Discussion}

LLD is a common orthopedic condition that arises from shortening or overgrowth of one or more bones in the lower limbs [3]. LLD occurs in about $10 \%$ of children in primary schools [9]. LLD $<2 \mathrm{~cm}$ is common and seldom causes a problem, but LLD $>3 \mathrm{~cm}$ causes distinct gait and
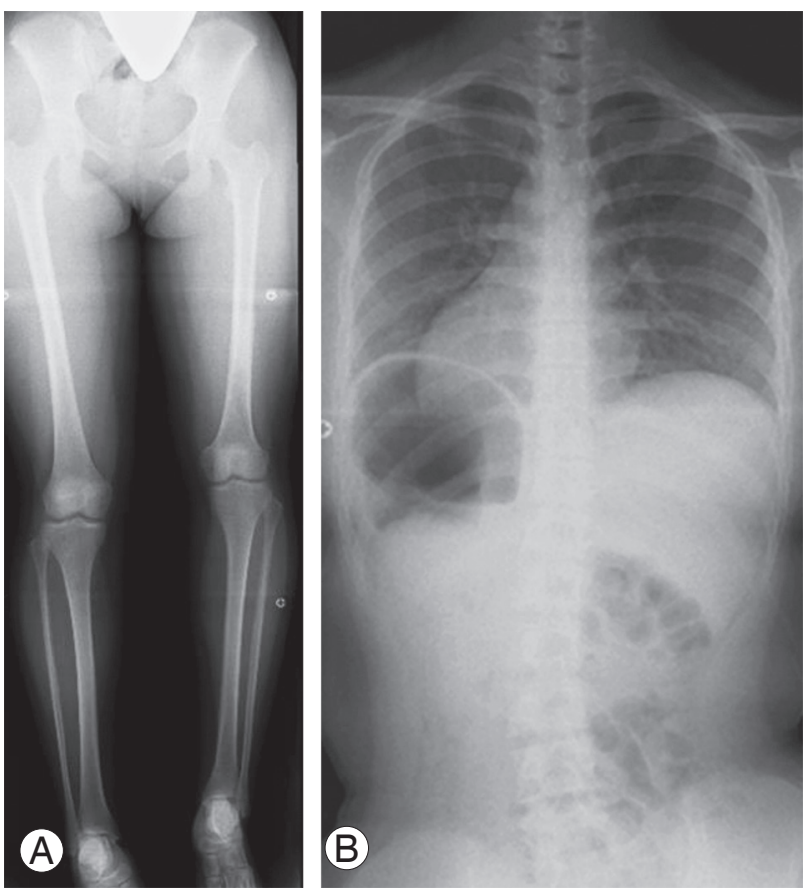

Fig. 5. Radiograph of case 1. (A) A 13-year-old female with left femoral hypoplasia and leg-length discrepancy $(51 \mathrm{~mm})$. (B) Pelvic obliquity was $16^{\circ}$, the Cobb angle was $21^{\circ}$ at Th12-L5, and the Nash/Moe index was 1.

Table 3. Details of the Cobb angle, pelvic oblique, and Nash/Moe index classified by LLD

\begin{tabular}{|c|c|c|c|c|c|}
\hline \multirow{2}{*}{ Variable } & \multicolumn{3}{|c|}{ LLD } & \multirow{2}{*}{$\begin{array}{c}p \text {-value } \\
\text { (ANOVA) }\end{array}$} & \multirow{2}{*}{ Post hoc test } \\
\hline & $20-39 \mathrm{~mm}^{\mathrm{a}}(\mathrm{n}=13)$ & $40-59 \mathrm{~mm}^{\mathrm{b}}(\mathrm{n}=5)$ & $\geq 60 \mathrm{~mm}^{\mathrm{c}}(\mathrm{n}=5)$ & & \\
\hline Major curve Cobb angle $\left(^{\circ}\right)$ & $9.5 \pm 4.0$ & $12.1 \pm 5.2$ & $22.8 \pm 5.4$ & $<0.05$ & $a<b<c$ \\
\hline Pelvic obliquity $\left({ }^{\circ}\right)$ & $4.6 \pm 1.3$ & $10.2 \pm 1.6$ & $19.0 \pm 6.2$ & $<0.05$ & $a<b<c$ \\
\hline Nash/Moe index of the apex vertebra $(n=0 / n \geq 1)$ & $12 / 1$ & $3 / 2$ & $2 / 3$ & $<0.05$ & $a<b, c$ \\
\hline
\end{tabular}

Values are presented as mean \pm standard deviation or number.

LLD, leg-length discrepancy; ANOVA, analysis of variance. 

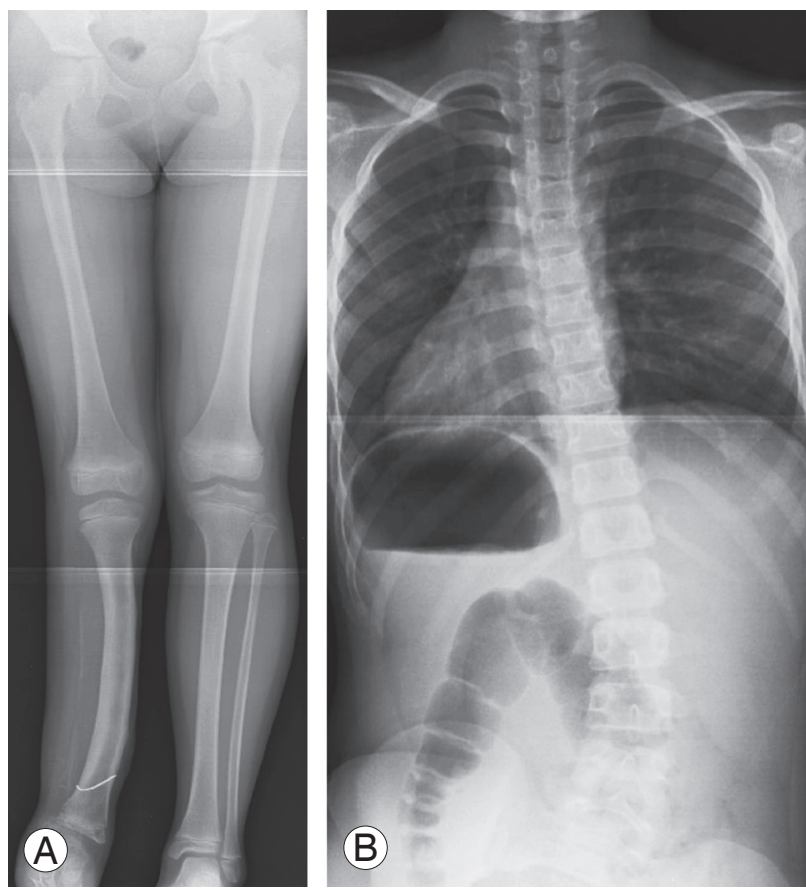

Fig. 6. Radiograph of case 2. (A) An 8-year-old female with congenital fibular hemimelia and leg-length discrepancy $(60 \mathrm{~mm})$. (B) Pelvic obliquity was $29^{\circ}$, the Cobb angle was $32^{\circ}$ at Th12-L5, and the Nash/Moe index was 1 .

posture disorders, and a larger LLD is associated with more distinct and severe disorders. Therefore, a greater LLD is not simply a cosmetic concern but also a functional concern due to postural imbalance when standing and an uneven gait.

LLD is caused by congenital or acquired abnormalities, such as growth arrest of the physis due to trauma or infection. In congenital LLD, the most common abnormalities are deficiencies of the femur, tibia, and fibula. Femoral disorders include proximal focal deficiency, congenital short femur, and hypoplastic femur. These are often accompanied by fibular hypoplasia or aplasia. Congenital unreduced dislocated hip and clubfoot also result in functional LLD [18]. Paralytic disorders, such as cerebral palsy, often result in growth inhibition due to reduced muscle activity and compression forces across the growth plate. Conditions that cause overgrowth of one side or part of the body include tumors and vascular malformations, such as hemangiomatosis and Klippel-Trenaunay-Weber syndrome. Stimulation is also seen with some nonvascular tumors, such as Wilms tumor, neurofibromatosis, and fibrous dysplasia. Conversely, tumors may inhibit growth through direct invasion of the growth plate or as a result of radiation used to treat the tumor. In acquired LLD, inflammation may alter bone growth; infection, juvenile rheumatoid arthritis, and hemophilia increase blood flow to the limb as part of the inflammatory process, thereby stimulating growth. Infection, particularly osteomyelitis, may also inhibit growth by destroying physeal cells. Trauma, most commonly due to fractures involving the physis, can lead to growth arrest, overgrowth, or angular deformity [3].

LLD can cause pelvic obliquity in the frontal plane [2,3,19-21], which may then lead to posture deformation, gait asymmetry, and discopathy; gonarthrosis, coxarthrosis, and hip flexion contracture on the longer side; and ankle joint contracture in the equinal position on the shorter side $[3,9,19]$.

Vink and Kamphuisen [22] suggested that scoliosis occurred with induced functional LLD, but spinal curves were not measured, and Caillet [23] stated that pelvic obliquity caused by an LLD $<0.75$ inches does not adversely affect scoliosis. In our series of LLD cases, the rate of scoliosis was $65 \%$, which is consistent with previous reports of $52 \%$ to $80 \%$ for the rate of spinal scoliosis associated with LLD $[8,10,24]$. The association of LLD and altered spinal biomechanics is demonstrated by the relationship between LLD and increased scoliosis. The Nash/ Moe index and vertebral body rotation were also significantly associated with LLD. In our study, there were no cases with a Nash/Moe index $\geq 2$, indicating the absence of cases with severe rotation. This may explain why there were no cases of severe scoliosis. Most scoliosis due to LLD is caused by a nonstructural property $[10,25]$, and in our series, there was a strong correlation between pelvic obliquity and the Cobb angle, which suggests that scoliosis reflects pelvic obliquity in standing due to LLD. Our results support a significant association between LLD and curvature of the spine [8]. However, it remains unclear whether improvement of scoliosis is possible using methods for LLD treatment, such as heel lift therapy. To date, there are insufficient studies of nonstructural scoliosis, and the interrelationships among LLD, tilt, pelvic rotation, and scoliosis require further investigation.

There are some limitations of the study. First, the number of cases is small, and the age of the subjects was variable. Thus, studies on a larger scale are needed. A sample size analysis using $G^{\star}$ Power software (ver. 3.1.3; HeinrichHeine-Universität Düsseldorf, Düsseldorf, Germany; http://www.gpower.hhu.de/). Our sample size analysis gave a statistical power for all groups of $75.2 \%$. A larger sample size was not essential because of the exploratory 
nature of the study, but we note that a statistical power over $80 \%$ is generally optimal for a significant result. Second, we only have fixed point measurements rather than longitudinal data. Giles and Taylor [26] reported case histories of scoliosis resolution after heel lift therapy for LLD $>5 \mathrm{~mm}$, but we did not evaluate the effect of neutralization or improvement of LLD on the spine or scoliosis. Third, we evaluated sagittal alignment using only whole spine PA standing radiographs, and three-dimensional (3D) imaging evaluation of leg length was not performed [27], although a biplanar low-dose X-ray device has been developed [11] for accurate 3D measurement of leg length [28]. However, the method of leg length measurement in our study gives the anatomical leg length and is not influenced greatly by position changes, distortion or magnification errors, parallax, pelvic conformation, or other geometric distortions, unlike previous evaluations using the height of the femoral head. We also note that there is no evidence that scoliosis is caused by congenital or acquired abnormalities that lead to LLD.

\section{Conclusions}

LLD is a common pediatric condition that can cause scoliosis of the spine. Severe scoliosis may develop if the LLD is $\geq 30 \mathrm{~mm}$. Long-term studies are needed to examine the effect of LLD resolution on the elimination of scoliosis.

\section{Conflict of Interest}

No potential conflict of interest relevant to this article was reported.

\section{Author Contributions}

$\mathrm{KK}$ analyzed data and wrote the manuscript; and $\mathrm{KA}, \mathrm{HN}$, MM, MM, SK, SI, TI, HY, KM, NI, and SI designed the study and collected data.

\section{References}

1. Gurney B. Leg length discrepancy. Gait Posture 2002;15:195-206.

2. Aaron AD, Eilert RE. Results of the Wagner and Ilizarov methods of limb-lengthening. J Bone Joint Surg Am 1996;78:20-9.

3. Rose R, Fuentes A, Hamel BJ, Dzialo CJ. Pediatric leg length discrepancy: causes and treatments. Orthop Nurs 1999;18:21-9.

4. Enjolras O, Chapot R, Merland JJ. Vascular anomalies and the growth of limbs: a review. J Pediatr Orthop B 2004;13:349-57.

5. Breugem CC, Maas M, Breugem SJ, Schaap GR, van der Horst CM. Vascular malformations of the lower limb with osseous involvement. J Bone Joint Surg Br 2003;85:399-405.

6. Goldstein WM, Gordon A, Branson JJ. Leg length inequality in total hip arthroplasty. Orthopedics 2005;28(9 Suppl):s1037-40.

7. Grzegorzewski A, Synder M, Kozłowski P, Szymczak W, Bowen RJ. Leg length discrepancy in Legg-CalvePerthes disease. J Pediatr Orthop 2005;25:206-9.

8. Specht DL, De Boer KF. Anatomical leg length inequality, scoliosis and lordotic curve in unselected clinic patients. J Manipulative Physiol Ther 1991;14:368-75.

9. Raczkowski JW, Daniszewska B, Zolynski K. Functional scoliosis caused by leg length discrepancy. Arch Med Sci 2010;6:393-8.

10. Papaioannou T, Stokes I, Kenwright J. Scoliosis associated with limb-length inequality. J Bone Joint Surg Am 1982;64:59-62.

11. Dubousset J, Charpak G, Dorion I, et al. A new 2D and 3D imaging approach to musculoskeletal physiology and pathology with low-dose radiation and the standing position: the EOS system. Bull Acad Natl Med 2005;189:287-300.

12. Young RS, Andrew PD, Cummings GS. Effect of simulating leg length inequality on pelvic torsion and trunk mobility. Gait Posture 2000;11:217-23.

13. Zabjek KF, Leroux MA, Coillard C, et al. Acute postural adaptations induced by a shoe lift in idiopathic scoliosis patients. Eur Spine J 2001;10:107-13.

14. Ploumis A, Trivedi V, Shin JH, Wood KB, Grottkau BE. Progression of idiopathic thoracic or thoracolumbar scoliosis and pelvic obliquity in adolescent patients with and without limb length discrepancy. Scoliosis Spinal Disord 2018;13:18.

15. Cobb JR. Outline for the study of scoliosis. Instr Course Lect AAOS 1948;5:261-75.

16. Sekiya T, Aota Y, Yamada K, Kaneko K, Ide M, Saito T. Evaluation of functional and structural leg length discrepancy in patients with adolescent idiopathic scoliosis using the EOS imaging system: a prospective 
comparative study. Scoliosis Spinal Disord 2018;13:7.

17. Nash CL Jr, Moe JH. A study of vertebral rotation. J Bone Joint Surg Am 1969;51:223-9.

18. Stanitski DF. Limb-length inequality: assessment and treatment options. J Am Acad Orthop Surg 1999;7:143-53.

19. Skwarcz A, Majcher P. Rehabilitation in scoliosis. In: Skwarcz A, Majcher P, editors. Medical rehabilitation. Wrocław: Urban \& Partner; 2003. p. 185-237.

20. Stricker SJ, Hunt T. Evaluation of leg length discrepancy in children. Int Pediatr 2004;19:134-46.

21. Walsh M, Connolly P, Jenkinson A, O’Brien T. Leg length discrepancy: an experimental study of compensatory changes in three dimensions using gait analysis. Gait Posture 2000;12:156-61.

22. Vink P, Kamphuisen HA. Leg length inequality, pelvic tilt and lumbar back muscle activity during standing. Clin Biomech (Bristol, Avon) 1989;4:115-7.
23. Caillet R. Scoliosis: diagnosis and management. Philadelphia (PA): FA Davis; 1975.

24. Fontanesi G, Giancecchi F, Rotini R. Segmental shortening and equalization for leg length discrepancies in adults. Ital J Orthop Traumatol 1987;13:45-54.

25. Moll JM, Liyanage SP, Wright V. An objective clinical method to measure lateral spinal flexion. Rheumatol Phys Med 1972;11:225-39.

26. Giles LG, Taylor JR. Low-back pain associated with leg length inequality. Spine (Phila Pa 1976) 1981;6:510-21.

27. Terry MA, Winell JJ, Green DW, et al. Measurement variance in limb length discrepancy: clinical and radiographic assessment of interobserver and intraobserver variability. J Pediatr Orthop 2005;25:197-201.

28. Gheno R, Nectoux E, Herbaux B, et al. Three-dimensional measurements of the lower extremity in children and adolescents using a low-dose biplanar X-ray device. Eur Radiol 2012;22:765-71. 